\title{
Gastrointestinal bleeding following NSAID ingestion in children
}

\author{
Lofty-John C. Anyanwu and Aminu M. Mohammad
}

\begin{abstract}
Gastrointestinal (GI) bleeding could result from various conditions in a child, and could be life threatening. Severe GI bleeds are, however, rare and their incidence is relatively unknown. Here, we report on two children who presented with GI bleeding following ingestion of NSAID. Two female children presented to our unit with GI bleeding. One was 6 years old and the other was 2 years old. Both presented with a history of fever and passage of bloody stools. There was a positive history of NSAID ingestion in both patients that was prescribed in the referring hospitals. The older child had a packed cell volume of $19 \%$ on presentation, and digital rectal examination indicated melaena. There was no history of vomiting. She received one unit of blood transfusion and was managed nonoperatively with antacids. Her recovery was uneventful. The younger patient presented with an abdominal distension and peritonitis, packed cell volume was $17 \%$ on presentation, and was malnourished.

Exploratory laparatomy showed two kissing perforations, one measuring about $2 \mathrm{~cm}$ in the second part of the
\end{abstract}

\section{Introduction}

Gastrointestinal (GI) bleeding resulting from various causes is common in children [1]. Severe life-threatening ones are, however, rare, and their incidence is relatively unknown.

NSAIDs are commonly used as antipyretic, analgesic, and anti-inflammatory agents. They are also used in cancer, Alzheimer's disease, and cardiovascular diseases [2]. These drugs are known to cause injury throughout the GI tract [1].

Elderly patients account for a large proportion of chronic users of NSAIDs [2]. The risk and the magnitude of NSAID-induced GI injury in children are uncertain. Here, we report two cases of GI bleeding in children following NSAID use.

\section{Case reports \\ Case 1}

A 6-year-old girl presented to our unit with a 1-week history of fever and passage of bloody stools for about $48 \mathrm{~h}$ before presentation. She had earlier been seen in a peripheral hospital, and was administered some medications that included Ibuprofen syrup. The patient had been on Ibuprofen for about 5 days before presentation, although the dosage administered could not be determined from the mother. She had no history of a similar bleeding.

Examination on presentation indicated a severely illlooking child who was febrile (temperature $38^{\circ} \mathrm{C}$ ), dehydrated, and pale. There were no peripheral stigmata of chronic liver disease. She was lethargic but conscious. duodenum and the other measuring $1 \mathrm{~cm}$ in the gallbladder. There were no demonstrable gallstones. She underwent a cholecystectomy and closure of the duodenal perforation. The patient, however, developed a superficial surgical site infection that responded to local wound care. Recovery was otherwise uneventful. A thorough assessment of drug history should be performed to rule out NSAID ingestion in a child with a GI bleed. Ann Pediatr Surg 9:87-89 (c) 2013 Annals of Pediatric Surgery.

Annals of Pediatric Surgery 2013, 9:87-89

Keywords: enteropathy, gastrointestinal-bleeding, NSAID

Paediatric Surgery Unit, Aminu Kano Teaching Hospital, Kano, Nigeria

Correspondence to Lofty-John C. Anyanwu, MBBS, MHPM, FWACS, Paediatric Surgery Unit, Aminu Kano Teaching Hospital, PO Box 2536 Kano, Kano-State, Nigeria

Tel: + 234803705 2659; e-mail: loftyjohnc@yahoo.com

Received 17 October 2011 accepted 23 February 2013

Her packed cell volume (PCV) was 19\% (PCV <30\% defined as anemia for the age group by our laboratory). Coagulation studies were not performed. A digital rectal examination showed melaena. There were no signs of peritonitis. The patient received an initial fluid resuscitation, followed by blood transfusion. An empirical antimalarial therapy was instituted on account of the pyrexia and oral antacids were administered. Her recovery was uneventful and she was discharged after 1 week of admission.

\section{Case 2}

A 2-year-old girl presented to us with a 10-day history of fever and a 2-day history of passage of bloody stools, with a progressive abdominal distension. She was initially seen in a peripheral hospital and was administered some medication that included Ibuprofen syrup. She was on the medication for about 1 week before presentation to our hospital. The dosage of the Ibuprofen could not be ascertained from her mother. She had no history of an abnormal bleed. Examination at presentation indicated a severely ill-looking child who was febrile (temperature $38.5^{\circ} \mathrm{C}$ ), pale, and anicteric. Her PCV was $17 \%$ (PCV $<30 \%$ defined as anemia for the age group by our laboratory). Coagulation studies were not performed. She was malnourished, with bilateral pitting pedal edema. Her abdomen was distended, and not moving with respiration, with a generalized tenderness. The examining finger was stained with blood following a digital rectal examination. An erect abdominal radiograph that included the lower chest showed air under the right hemi diaphragm. She underwent an emergency laparatomy 
following resuscitation and blood transfusion. The findings at surgery were two kissing perforations, one measuring about $2 \mathrm{~cm}$ in diameter in the second part of the duodenum and the other measuring $1 \mathrm{~cm}$ in diameter in the gallbladder (Fig. 1). There were no demonstrable gallstones. The duodenal perforation was closed and she underwent a cholecystectomy. The patient received a course of postoperative antibiotics and antacids. She, however, developed a surgical site infection that responded to local care. Nutritional rehabilitation was instituted, and following an improved clinical condition, she was discharged home after 40 days of admission.

\section{Discussion}

GI injury is a major adverse effect of NSAID use. The use of these drugs is known to disrupt GI-protective mechanisms, which include mucosal blood flow and mucus secretion [3]. These disruptions lead to an impairment in the ability of the mucosa to respond to or to resist injury. The suppression of mucosal prostaglandin synthesis, by the systemic inhibition of cyclooxygenase (COX) enzymes, confers on NSAIDs their ability to injure the GI mucosa [2-4]. NSAIDs, being weak acids, also cause topical mucosal irritation and damage by various mechanisms [2].

It is estimated that in the USA, about 100000 hospitalizations occur each year for serious NSAIDassociated GI complications, with an annual mortality of about 16500 patients [5].

There is a paucity of data on the incidence of these injuries in the pediatric population. In our environment, NSAID syrups are readily available over the counter drugs

\section{Fig. 1}

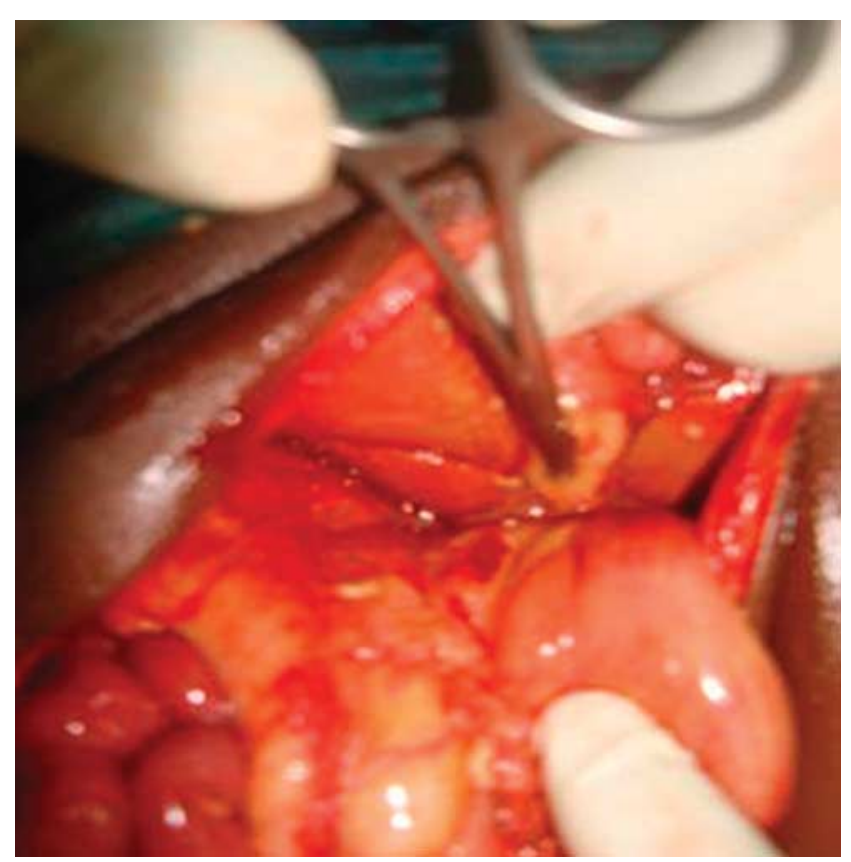

Intraoperative image showing gallbladder perforation (artery forceps as a pointer). Opposite is duodenal perforation. that some use as antipyretic or analgesic agents. Both of our patients had taken Ibuprofen syrup prescribed in peripheral hospitals before the onset of their GI bleeding.

Different NSAIDs do show different risks for GI ulceration [4]. Studies in Denmark have shown that for Ibuprofen and Naproxen, there was a clear trend in an increasing risk for upper GI bleeding with increasing dose [6].

It is believed that NSAID-induced enteropathy is more common than injuries of the stomach and duodenum, manifesting usually as occult blood loss or hypoalbuminemia [5,7]. The mechanisms responsible for NSAIDinduced enteropathy have not been fully understood $[4,5]$. Topical irritation by NSAIDs appear to play a role here, given that enterohepatic recirculation of these drugs prolongs the exposure of the intestinal mucosa to them, with the luminal bile and enterobacteria potentiating the damage after the initial increase in gut permeability induced by the NSAID [3,4]. Parenterally administered NSAIDs could also lead to intestinal mucosal injury, as it has been shown in experiments in rats that subcutaneously administered indomethacin caused jejunal and ileal mucosal damages [3]. Systemic inhibition of COX-1 and COX-2 enzymes may be involved [5].

Systemic inhibition of COX-1 is believed to lead to the release of endothelin-1 a potent vasoconstrictor, which may induce mucosal injury by the reduction of blood flow [8]. Sustained vasoconstriction worsens mucosal injuries, causing extensive necrosis and hemorrhage $[4,8]$. In the adult population, the risk of progression of NSAID-induced ulcers to the development of bowel perforation or excessive hemorrhage in chronic NSAID users is $2-4 \%$ [4].

It has been shown in animal experiments that there is an age-dependent variation in intestinal injury $[9,10]$. Both of our patients were children who developed GI bleeding following NSAID use.

Nitric oxide (NO), a vasodilator, is believed to mediate certain physiological functions in the GI tract that include mucosal blood flow, maintenance of mucosal integrity, and maintenance of vascular tone [3]. The inducible NO synthase (iNOS), one of the three isoforms of NO synthase, is characterized by its ability to produce high quantities of NO [9]. It has been shown that iNOS activation in response to lipopolysaccharide stimulation in the ileum of rats varies with age, being 10 -fold higher in adolescents than in neonates [3,9]. Gosche et al. [10] reported that in rats, the neonatal intestine had a reduced compensatory response to hemorrhage, hypothermia, and hypoxia compared with the adult's. Some believe that reduced iNOS induction may explain why the intestinal microvasculature in the immature rats lacks vasodilator responses that are active in the mature rats [9].

Studies have shown that most GI injuries following NSAID use would commonly occur within the first 30 days following the commencement of the NSAID [11]. Both of our patients had been on Ibuprofen for about 1 week before the onset of bloody stools. 
Our second patient presented with a duodenal perforation together with a gallbladder perforation (Fig. 1). We were unable to ascertain whether one was related to the other or whether they were both independent injuries resulting from NSAID use. We are not aware of any report in the literature of a gallbladder perforation induced by NSAID use. This report of a gallbladder perforation following NSAID use may be an unexplored topic for NSAID-associated injury, given the role of enterohepatic circulation in concentrating these drugs in the biliary system [4,5]. Endoscopic studies could not be performed in these patients because of the non availability of this facility in our institution.

NSAID enteropathy may produce a higher disease burden in children, given that there are age-related differences in the effectiveness of intestinal blood flow autoregulation [10]. The mucosal epithelium is believed to be the primary barrier against bacterial translocation in the intestine, [9] and injuries to this barrier will allow gut bacterial translocation, leading to septicemia, and may also manifest as NSAID enteropathy, resulting in occult blood loss and hypoalbuminemia [7].

The prolonged duration of admission (40 days) in our second patient was because of the long period of nutritional rehabilitation she had to undergo. This long duration of admission constituted an enormous financial burden to her parents. Her malnourished state also contributed toward her poor wound outcome.

We recommend that a high index of suspicion should be adopted in the evaluation and management of any child with a GI bleed, and that a thorough assessment of drug history should be carried out to rule out NSAID ingestion.

\section{Acknowledgements}

\section{Conflicts of interest}

There are no conflicts of interest.

\section{References}

1 Boyle JT. Gastrointestinal bleeding in infants and children. Pediatr Rev 2008; 29:39-52.

2 Al Mofleh IA, Al Rashed RS. Nonsteroidal anti-inflammatory drug induced gastrointestinal injuries and related adverse reactions: epidemiology, pathogenesis and management. Saudi J Gastroenterol 2007; 13: 107-113.

3 Lanas A. Role of nitric oxide in the gastrointestinal tract. Arthritis Res Ther 2008; 10:S4 [Epub ahead of print].

4 Perini R, Fiorucci S, Wallace JL. Mechanisms of nonsteroidal antiinflammatory drug induced gastrointestinal injury and repair: a window of opportunity for cyclooxygenase inhibiting nitric oxide donors. Can $J$ Gastroenterol 2004; 18:229-236.

5 Ramirez-Alcantara V, LoGuidice A, Boelsterli UA. Protection from diclofenacinduced small intestinal injury by the JNK inhibitor SP600125 in a mouse model of NSAID-associated enteropathy. Am J Physiol Gastrointest Liver Physiol 2009; 297:G990-G998.

6 Mellemkjaer L, Blot WJ, Sorensen HT, Thomassen L, McLaughlin JK, Nielsen GL, Olsen JH. Upper gastrointestinal bleeding among users of NSAIDs: a population-based cohort study in Denmark. Br J Clin Pharmacol 2002; 53:173-181.

7 Kim JW. NSAID-induced gastroenteropathy. Korean J Gastroentero/ 2008; 52:134-141.

8 Wallace JL. Prostaglandins, NSAIDs, and gastric mucosal protection: why doesn't the stomach digest itself? Physiol Rev 2008; 88: 1547-1565.

9 Morin MJ, Karr SM, Faris RA, Gruppuso PA. Developmental variability in expression and regulation of inducible nitric oxide synthase in rat intestine. Am J Physiol Gastrointest Liver Physiol 2001; 281: G552-G559.

10 Gosche JR, Harris PD, Garrison RN. Age related differences in intestinal microvascular responses to low-flow states in adult and suckling rats. Am J Physiol Gastrointest Liver Physiol 1993; 264:G447-G453.

11 Berezin SH, Bostwick HE, Halata MS, Feerick J, Newman LJ, Medow MS Gastrointestinal bleeding in children following ingestion of low-dose Ibuprofen. J Pediatr Gastroenterol Nutr 2007; 44:506-508. 